\title{
Optimization and Cold Test of a Triaxial 2G HTS Power Cable with High Current Capacity
}

\author{
S.S. Fetisov, V.V. Zubko, S.Yu. Zanegin, A.A.Nosov and V.S. Vysotsky, Senior Member IEEE
}

\begin{abstract}
Triaxial HTS AC power cables, that incorporates three HTS phases wound around a single core within a single cable is considering the optimal solution for low and medium voltages. Such a design permits to save expensive HTS conductor and to increase the power density transmitted. It is difficult to increase the transmission capacity of a triaxial cable by increasing its operating voltage and therefore, insulation thickness between phases. The other way is to increase the operation current by using a multilayer structure in each phase of the triaxial cable. This task demands a complicated optimization analysis and a precise manufacturing technology. Following our previous studies, we developed and tested the triaxial cable prototype with two layers per phase made of ReBCO wire. The optimal parameters of the cable (twist pitch, diameters of phases, etc.) have been determined using the numerical simulation methods developed. DC and AC tests of the cable has been performed. In the result, the feasibility to provide a uniform current distribution in a triaxial cable, each phase of which consists of two layers, has been demonstrated. With an outer diameter of $\sim 24 \mathrm{~mm}$ only, this is the most compact triaxial HTS power cable manufactured and tested up to date with currents up to $4 \mathrm{kA}$ per phase. The anticipated power transfer with three phases opf this cable can be as large as $\sim 34 \mathrm{MW}$.
\end{abstract}

Index Terms - HTS cables, Triaxial cables, 3D FEM modelling

\section{INTRODUCTION}

$\mathbf{H}$ igh temperature superconducting (HTS) power cables are the most advanced application of superconductivity in the electric power industry, many HTS power cables have already been installed in power grids and have been successfully tested [1],[2].

Along with usual the "single core" or "three-in-one" designs [2] that are using coaxial HTS cables where each phase has its own core and shield, the power HTS cables with a triaxial design are of a great interest [2].

A triaxial (HTS) AC power cable incorporates three HTS phases wound around a single core within a single cable. It is the optimal solution for low and medium voltages. This design permits to save half of expensive HTS wires and to increase

Manuscript receipt and acceptance dates will be inserted here.

This work is supported by the Russian Scientific Foundation under Grant №16-19-10563П. (All authors contributed equally to this work.) (Corresponding author: Vitaly Vysotsky, e-mail: vysotsky@ieee.org).

S.S. Fetisov, V.V. Zubko, S.Yu. Zanegin, A.A. Nosov and V.S. Vysotsky are with Russian Scientific R\&D Cable Institute, 111024. Moscow, Russia.

V.S. Vysotsky is also with National Research Nuclear University MEPhI (Moscow Engineering Physics Institute), 115409, Moscow, Russia. (e-mail: vysotsky@ieee.org).

Digital Object Identifier will be inserted here upon acceptance. the power density transmitted. This is because all three phases should be balanced and a current in an outer shield is zero. Therefore, such a shield could be made of copper and does not need expensive HTS conductor. The copper shield in a triaxial cable is necessary for the protection of all the current-carrying phases in case of a fault.

The advantages of triaxial cables were proved by the largescale Bixby and AmpaCity projects [3], [4]. These triaxial cables were designed with use of one HTS layer per each phase made of $1 \mathrm{G} \mathrm{Bi}-2223$ tapes.

In addition to the already implemented projects with "threein-one" designs of HTS cables [5], the research activities are carrying out in South Korea to develop triaxial cables. For example, the triaxial $23 \mathrm{kV} / 60$ MVA cable where one phase (A) has two layers [6] has been studied.

Two triaxial cables prototypes have been developed and tested in Russian Scientific R\&D Cable Institute [7]. Along with $10 \mathrm{~m} \mathrm{Bi}-2223$ triaxial cable with one HTS layer, the one layer per phase triaxial cable made of ReBCO HTS wires have been developed [7] as well. This cable has been successfully tested and demonstrated about 20 times less AC losses than the power cable with the same parameters made of $1 \mathrm{G}$ HTS wires [7].

It is difficult to increase the transmission capacity of a triaxial cable by increasing its operating voltage and, therefore, an insulation thickness between phases. Increase of insulation thickness leads to increasing a diameter of a cable that is undesirable as this will greatly reduce the flexibility of the cable. That is why the maximum voltage between coaxial phase in a triaxial cable probably will not exceed $\sim 20 \mathrm{kV}$.

To increase the power transmitted by a triaxial with keeping a cable diameter small enough, the other way could be proposed: increasing an operation current by using a multilayer structure in each phase of a cable. This task demands a complicated optimization analysis to provide the proper current distribution between phases and tape layers in each phase.

In Russian Scientific R\&D Cable Institute the research project is underway aimed on reducing as much as possible mass and dimensions of HTS power cables. This is important, for example, for electrical aircrafts; ship propulsion systems and other applications were sizes and mass are critical. The feasibility of compact cables with a small diameter became possible with development of advanced ReBCO 2G HTS wires that allow smaller bending diameter without current degradation. The reduction of cables diameters allows the lower cables 
bending diameter also that is also important for the applications with limited space.

In this paper we present the results of development and tests of the compact triaxial HTS power cable having two HTS layers per phase. Our major goals were development and substantiation of optimization method, as well as the development of a production technology that ensures a uniform current distribution between the phases of the cable and between layers in each phase.

Optimization of the current distribution between layers of the cable phases was carried out using two numerical models. The first model uses a cable electrical circuit with current sources. The second one, detailed three-dimensional (3D) model, uses the finite element method (ANSYS Emag). $\sim 4 \mathrm{~m}$ long cable prototype has been made and its electrical tests were carried out. As a result, the feasibility of obtaining the uniform current distribution has been demonstrated in a compact triaxial HTS cable, each phase of which consists of two layers.

\section{Numerical Models to Design the Multilayer TriaX- IAL HTS CABLE}

As in our previous works with single core compact coaxial cables [8]-[10], the numerical models have been developed. The first one is based on an equivalent electrical circuit and the second one on the three-dimensional (3D FEM) model that uses the finite element method to optimize the distribution of currents between layers in the phases of the triaxial cable,

For optimization of the current- carrying element of a triaxial three phase HTS cable on the base of equivalent electrical circuit we used the standard mathematic equations allowing calculations of currents and voltages in phases:

$$
\begin{aligned}
& \frac{\partial U_{k}(t, z)}{\partial z}=R_{k}\left(I_{k}\right) I_{k}(t)+\sum_{i=1}^{N_{p}}\left\{\frac{d I_{k, i}}{d t}\left(M_{k, i}\right)\right\} \\
& \frac{\partial I_{k}(t, z)}{\partial z}=\sum_{i=1}^{N_{p}}\left\{\frac{d U_{k, i}}{d t}\left(C_{k, i}\right)\right\}
\end{aligned}
$$

where $N$-number of layers; $M_{k}$ и $M_{k i}$ - self-inductances of layers and mutual-inductances between layers; $I_{k}, U_{k}$ and $d I_{k} / d t$ - current, voltage and magnitude of a current change in time in each layer correspondingly; $R\left(I_{k}\right)$ - resistance of a layer dependent on a current; $C_{k i^{-}}$electrical capacitance between layers.

In the multilayer triaxial HTS cable the maximum current values in the layers of each phase should be equal to each other, for example, for phase A:

$I_{A}=\sum_{i=1}^{N} I_{A, i}, \quad I_{A, i}=I_{A, i+1}=\frac{I_{A}}{N}$

The problem of determining the uniform distribution of currents between the layers in each phase of the cable can be formulated as:
$F(X)=\min f(X)=$

$\sum_{k=1}^{m-1} \sum_{i=k+1}^{m}\left|I_{k}(X)-I_{i}(X)\right|+\sum_{k=m+1}^{N-1} \sum_{i=k+1}^{N}\left|I_{k}(X)-I_{i}(X)\right|$.

where $I_{k}$ - the maximum values of currents in the layers obtained from numerical models, - vector of the optimized variables, $r_{k}$ - inner radius of layers, $a_{k}$ is winding direction of HTS tapes in a layer, $L_{k}$ is the twist pitch of the $k$ layer.

In addition, in order to balance the currents and voltages in the phases $(A, B, C)$ of the cable, their values must satisfy the following conditions:

$I_{B}=\beta^{2} I_{A}, \quad I_{C}=\beta I_{A}$,

$V_{B}=\beta^{2} V_{A}, \quad V_{C}=\beta V_{A}, \quad \beta=\exp (j 2 \pi / 3)$

where $j$ is the imaginary unit.

Two FEM models have been also developed using the ANSYS Emag software for complex study of the triaxial 2G HTS cable.

For faster calculations the 3D FEM model with simpler geometrical configuration has been developed, in which layers in the phases of the cable are modeled by a system of thin concentric layers (cylinders) where all the layers are isolated from each other [8]. In superconducting thin cylinders, the optimized variables (the winding direction and twist pitch of the HTS tapes) are modeled by the anisotropy of the electrical conductivity of each cylinder.

The detailed 3D FEM model was also used to verify the result of the optimization. This model offers the possibility to modelling any topology of the HTS cable. The model allows considering the spiral structure of the layers to obtain current and detailed magnetic field distributions inside the cable.

In Fig. 1, the mesh elements in detailed 3D model of the HTS tape layers is shown on the end part of the triaxial cable. The cable contains two layers in each phase.

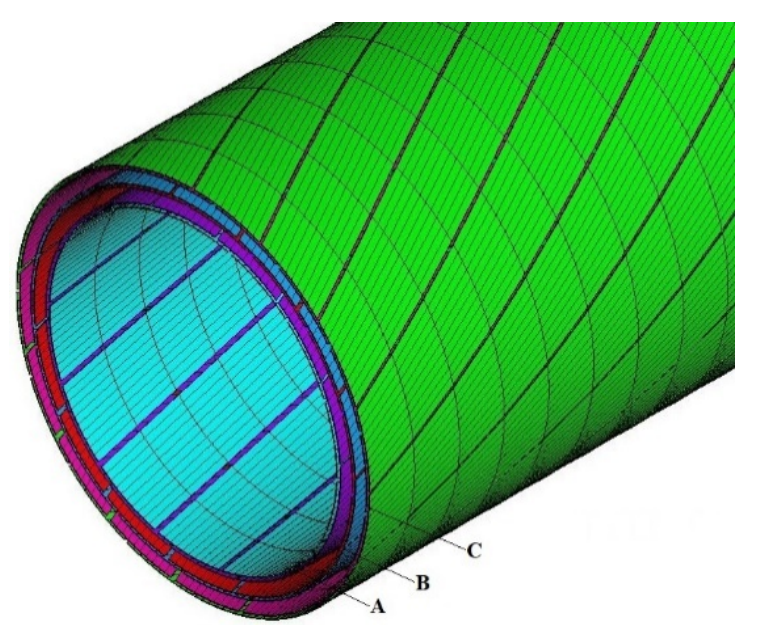

Fig. 1. The elements of the HTS tape layers on the end part of the cable (2layers per phase) in detailed 3D FEM model. Letters A, B, C indicate the phases of the cable. 


\section{DeVelopment of the TriaXial Cable with Two Lay- ERS PER PHASE}

\section{A. The HTS Tapes Used}

To manufacture the triaxial cable prototype with two layers per phase, we used the ReBCO 2G HTS tape produced by SuperOx with a total thickness of $\sim 0.105 \mathrm{~mm}$ and a width of $4 \mathrm{~mm}$. The critical currents of these tapes in self-field at a temperature of $77.4 \mathrm{~K}$ were $\sim 130-150 \mathrm{~A}$.

\section{B. Optimization Results}

We performed optimization using the equivalent electrical circuit of the cable, and then these results were compared with the calculations by the 3D FEM models.

The final parameters of optimized cable are listed in Table 1. The sign in the twist pitch column indicates the direction of tapes twisting. To ensure minimum AC losses, it is necessary to provide the smallest possible gaps between the tapes in the cable layers.

TABLE I

\begin{tabular}{ccccc}
\multicolumn{5}{c}{ PARAMETERS OF THE MODEL CABLE DESIGN } \\
\hline \hline Phase & $\begin{array}{c}\text { Number of } \\
\text { the layer }\end{array}$ & $\begin{array}{c}\mathrm{D} \text { min, } \\
\mathrm{mm}\end{array}$ & $\begin{array}{c}\text { Twist pitch, } \\
\mathrm{mm}\end{array}$ & Number of tapes \\
\hline \multirow{3}{*}{ A inner } & 1 & 19.3 & 324 & 14 \\
& 2 & 19.8 & -171 & 14 \\
B middle & 3 & 21.4 & 200 & 15 \\
& 4 & 21.8 & -161 & 15 \\
C outer & 5 & 23.35 & 191 & 16 \\
\hline
\end{tabular}

As an example, in Fig. 2 the magnetic fields in the cable are shown, calculated by the FEM model for two times $(0.005 \mathrm{sec}$ and $0.01 \mathrm{sec}$ ). One can see that there is no magnetic field outside the triaxial cable. That is why triaxial cables do not need outer superconducting shields. It means that three phase triaxial cables need two times less superconducting wires in comparison with usual three single-phase coaxial cables.

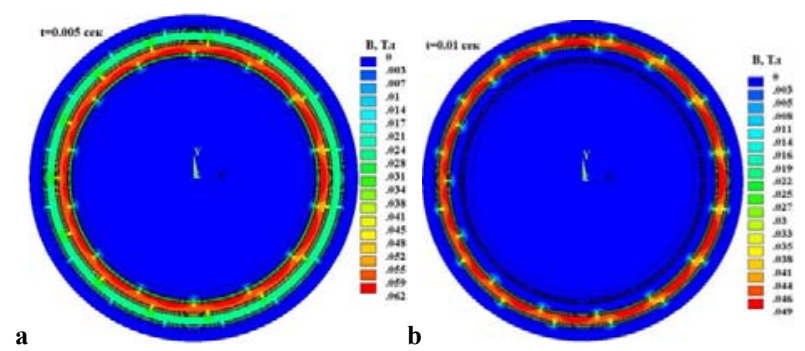

Fig. 2. The magnetic fields in the triaxial cable $: \mathrm{a}-\mathrm{t}=0.005, \mathrm{~b}-\mathrm{t}=0.01 \mathrm{~s}$.

To obtain a uniform current distribution in a compact multilayer cable, the precise winding of layers should be provided. The method of the precise winding has been developed in our previous works [8]-[9]. During cable manufacturing, after winding of each layer, its average diameter has been measured, then the parameters of the following layers were recalculated to be optimized. This method allowed us to obtain a precise cables layers diameters and twist pitches.
It is also necessary to have a tough (but flexible) former and tightly wound insulation that do not change their diameters when HTS tapes are applied over them. In our cable prototype the former is the central supporting element consisting of tightly twisted copper wires, is playing the role of the main strength carrying element when installing (pulling) the cable into the cryostat, and also it performs the function of an electric shunt in emergency operating modes (faults) of the HTS cable. The former is covered with polyimide tape to insulate it and to level the surface. All of above eventually allowed us to obtain uniform current distribution in the triaxial cable prototype.

In Fig. 3 the prototype cable assembled for the test and view of the test are shown.

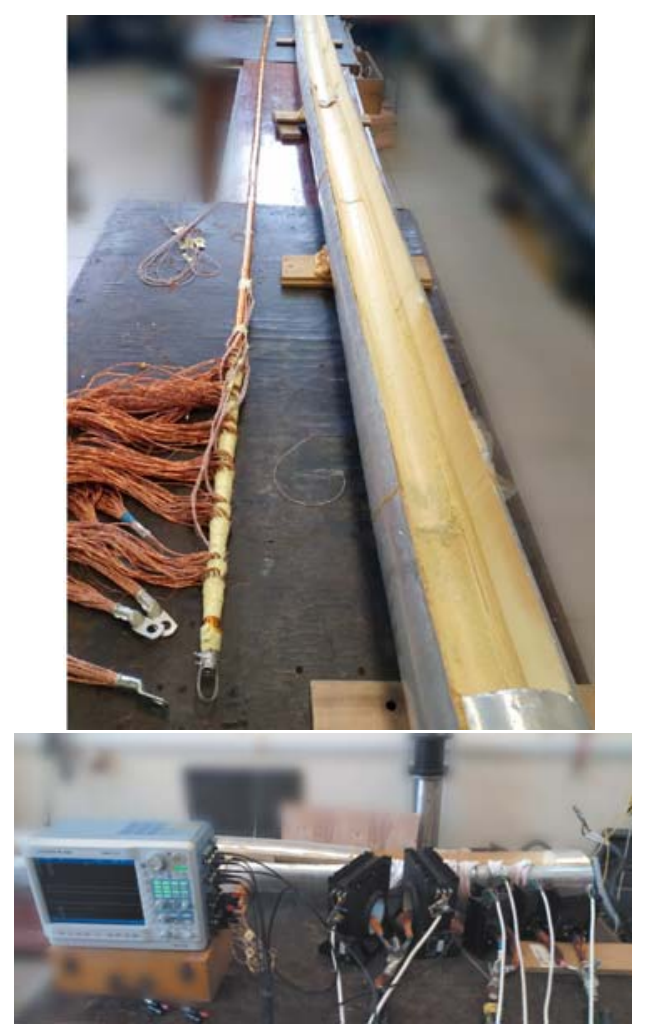

Fig.3. Top: triaxial cable prototype assembled for the test and its test cryostat, bottom: data acquisition system and the cable during tests.

\section{EXPERIMENTAL RESULTS}

The standard test program for cables in Russian Scientific R\&D Cable institute includes (but not exhausted):

-DC test to determine critical currents in each layer/phase;

-AC test to determine current distribution between layers at AC conditions;

The test methods were earlier described in details [7], [11].

\section{A. DC Tests}

Critical currents of the cable layers $\left(I_{c}\right)$ were determined by measuring the voltage-current characteristic for each layer according to the criterion of $1 \mu \mathrm{V} / \mathrm{cm}$ at the temperature of $77.4 \mathrm{~K}$. The following values of $I_{c}$ were obtained: the first lay- 
er -1969 A; the second layer - 2070 A, total in phase A - 4039 $\mathrm{A}$; third layer - $2192 \mathrm{~A}$; the fourth layer - $2011 \mathrm{~A}$, total in phase B - $4203 \mathrm{~A}$; the fifth layer -2003 A; the sixth layer 2016 A, total in phase C - 4019 A. The experimental values of critical currents in the layers are close to the expected from sum of currents of all tapes in a layer. It means that three phase triaxial cables need two times less superconducting wires in comparison with usual three single-phase coaxial cables.

We also can conclude that during manufacturing of the cable there is no degradations of critical currents in the HTS tapes due to mechanical deformation (bending of the tapes when they are placed to layers).

\section{B. The Distribution of Currents between Layers}

Fig. 4 shows the measured currents in the phases of this model cable at a frequency of $50 \mathrm{~Hz}$. The maximum currents in the layers of phases practically coincide. However, there is a slight shift between the currents of the layers of each phase (about 5-8 degrees in relation to the optimal values).

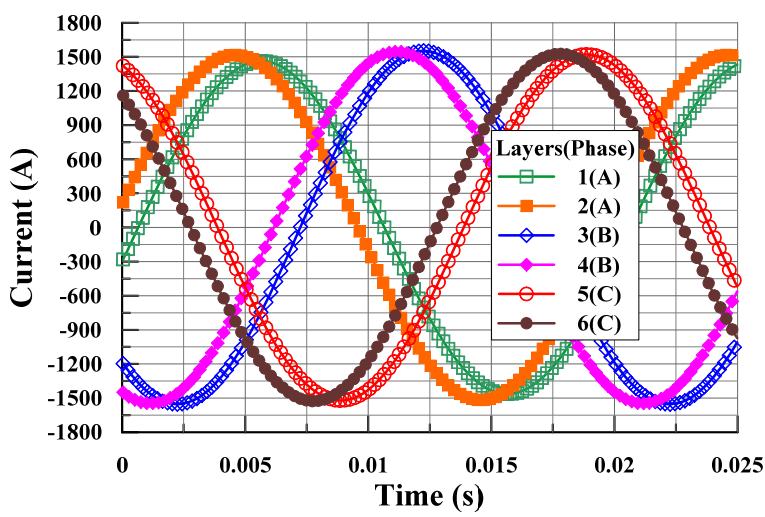

Fig. 4. The measured currents in the phases of the triaxial cable at $50 \mathrm{~Hz}$ frequency.

The measurements of currents were carried out at different frequencies. Fig. 5 demonstrates the ratio of the current in the inner layer to the current in the outer layer for all phases of the cable versus frequency. One can see that by using our optimization methods and manufacturing technology. we managed to achieve almost uniform current distribution among the layers in phases. Small deviations from uniformity at maximum currents and phase shifts are most likely associated with the influence of current leads (terminations).

\section{CONCLUSION}

The optimized design and technology have been developed for manufacturing a compact triaxial cable containing two layers in each phase made of advanced 2G ReBCO HTS tapes.

Optimization of the current distribution between the layers in the cable phases was carried out using three numerical models. The first model uses a cable circuit with current sources. Other two detailed 3D models, use the finite element method.
A prototype $4 \mathrm{~m}$ long cable was fabricated and tested both at $\mathrm{DC}$ and $\mathrm{AC}$ current modes.

In the result, the feasibility has been demonstrated of obtaining the uniform current distribution in a triaxial cable, each phase of which consists of two layers,.

With an outer diameter of $\sim 24 \mathrm{~mm}$, this is the most compact triaxial HTS power cable manufactured and tested up to date with currents up to $\sim 4 \mathrm{kA}$ per phase.

With insulation thickness phases $\sim 0.8 \mathrm{~mm}$ (A-B) and $\sim 1 \mathrm{~mm}$ (B-C) we anticipate operation voltage $\sim 5 \mathrm{kV}$ (test voltage $\sim 10 \mathrm{kV}$ ). That means our small cable could transfer $\sim 34 \mathrm{MW}$ AC electric power via its three phases.

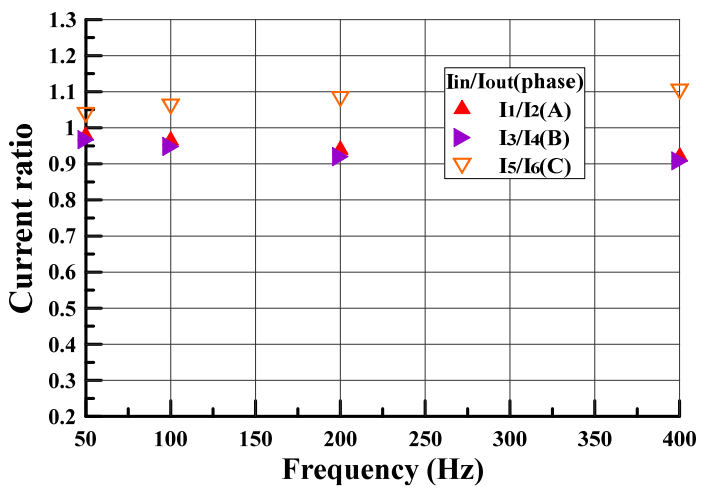

Fig. 5. The ratio of the maximum current in the inner layer of each phase to the current in the outer layer depending on the frequency.

\section{REFERENCES}

[1] D.I. Doukas, "Superconducting Transmission Systems: Review, Classification and Technology Readiness Assessment," IEEE Trans. on Appl. Supercond., vol. 29, no. 5, 2019, Art. no. 5401205.

[2] A.P Malozemoff, J. Yuan, C.M. Rey, "High-temperature superconducting (HTS) AC cables for power grid applications. Superconductors in the Power Grid," Woodhead Publishing, 2015, p. 133-188.

[3] J.A. Demko, I. Sauers, D. R. James, M.J. Gouge, D. Lindsay, M. Roden, J. Tolbert, D. Willén, C. Træholt, and C.T. Nielsen, "Triaxial HTS Cable for the AEP Bixby Project," IEEE Trans. Appl. Supercond., vol. 17, no. 2, 2007, p. 2047-2050.

[4] M. Stemmle, F. Merschel, M. Noe, A. Hobl, "AmpaCity - Advanced superconducting medium voltage system for urban area power supply," T\&D Conference and Exposition, 2014 IEEE PES, 2014, pp. 1-5.

[5] C. Lee, H. Son, Y. Won, Y. Kim, et al, "Progress of the first commercial project of high-temperature superconducting cables by KEPCO in Korea," Supercond. Sci. Technol., vol. 33, no. 4, 2020, Art. no.044006.

[6] S. Lee, S. Kang, M. Park, D. Won, J. Yoo, H. Yang, "Performance Analysis of Real-Scale 23 kV/60 MVA Class Tri-Axial HTS Power Cable for Real-Grid Application in Korea," Energies, vol. 13, no. 8, 2020, 2053.

[7] S.S. Fetisov, V.V. Zubko, S.Y. Zanegin, A.A. Nosov, S.M. Ryabov and V.S. Vysotsky, "Study of the First Russian Triaxial HTS Cable Prototypes," IEEE Trans. Appl. Supercond., vol. 27, no. 4, 2017, Art. no. 5400305 .

[8] S. Fetisov, V. Zubko, S. Zanegin, A. Nosov, V. Vysotsky "Numerical Simulation and Cold Test of a Compact 2G HTS Power Cable," IEEE Trans. on Appl. Supercond., vol. 28, no 4, 2018, Art. no. 5400905.

[9] S.S. Fetisov, V.V. Zubko, S.Y. Zanegin and V.S. Vysotsky, "Cold test and numerical analysis of the compact $2 \mathrm{G}$ HTS power cable," Conf. Series: Materials Science and Eng. vol. 502, no. 1, 2019, 012179.

[10] S S Fetisov, V V Zubko, S Yu Zanegin, A A Nosov and V S Vysotsky Compact 2G HTS power cable: new cold tests results, Journal of Physics: Conference Series, 1559, 2020, Art no. 012081.

[11] V.S. Vysotsky, A. A. Nosov, S. S. Fetisov, K.A. Shutov, AC Loss and Other Researches with 5 m HTS Model Cables, IEEE Transactions on Applied Superconductivity, Vol.21, N3, 2011, pp.1001-1004. 